\title{
Versorgungsstrukturgesetz:
}

\section{Was bringt die Reform der Honorarreform?}

\section{Stephan Feldmann}

Dipl. Volkswirt Stephan Feldmann ist Vertragsreferent Ärzte beim AOK-Bundesverband

\begin{abstract}
Das Pendel schlägt zurück. Nach wenigen Jahren der Zentralisierung und der Versuche, Vergütungen und Honorare bundeseinheitlich zu regeln, sieht der Kabinettsentwurf zum Versorgungsstrukturgesetz eine umfassende Re-Regionalisierung der Vergütungs- und Honorarpolitik vor. Hatte der Gesetzgeber noch mit dem GKV-Finanzierungsgesetz die Selbstverwaltung auf Bundesebene beauftragt, mit der Erarbeitung einer Konzepts zur Konvergenz der Vergütungen einen Beitrag zur Überprüfung der Honorarreform zu leisten, haben die sich daran anschließenden Diskussionen und die sich dabei entwickelnden innerärztlichen Gegenkräfte gegen eine Neu- und Umverteilung das Ende der Zentralisierung herbeigeführt. Die Politik mischt die Karten neu und hat eine umfassende Kompetenzrochade auf den Weg gebracht. Im Folgenden sollen die grundsätzlichen Weichenstellungen des Kabinettsentwurfs zum Versorgungsstrukturgesetz hinsichtlich der vertragsärztlichen Vergütung analysiert und ein Ausblick gegeben werden.
\end{abstract}

\section{Die Ausgangslage nach dem Wettbewerbsstärkungsgesetz}

Das kollektivvertragliche ambulante ärztliche Vergütungssystem war zuletzt in den Jahren 2008/2009 auf Basis der Rahmenvorgaben des GKV-Wettbewerbsstärkungsgesetzes (GKV-WSG) umfassend reformiert worden. Übergeordnetes Ziel dieser grundlegenden Neuausrichtung war es, mehr Transparenz - insbesondere für die Leistungserbringer in Form fester Preise - zu verbessern und die bestehenden weder begründbaren noch zielführenden Vergütungsunterschiede sowohl zwischen den Ärzten als auch zwischen den Kassen aufzuheben. Rund eineinhalb Jahrzehnte waren die von den Krankenkassen an die Kassenärztlichen Vereinigungen (KVen) entrichteten Gesamtvergütungen entsprechend des jährlichen Grundlohnsummenanstiegs fortgeschrieben worden, dies unabhängig von Veränderungen der Versichertenstrukturen,
Leistungsinanspruchnahmemuster und Angebotsstrukturen. Dies hatte mit der Zeit zu erheblichen Ungleichgewichten zwischen den gezahlten kassenspezifischen Gesamtvergütungen und den tatsächlich von den jeweiligen Versicherten einer Kasse in Anspruch genommenen Leistungen geführt. Regionale Unterschiede hinsichtlich der Abgrenzung inner- und außerbudgetärer Leistungen sowie bundesweite Uneinheitlichkeit bei der Implementierung von Mengensteuerungsmechanismen auf Ebene der arztseitigen Honorarverteilung trugen zusätzlich dazu bei, dass sowohl auf regionaler als auch Kassenebene für gleiche Leistung fast unübersehbar viele Preise gezahlt wurden, ohne dass dies systematisch (z.B. durch unterschiedliche Kostenstrukturen) begründet gewesen wäre und nur in wenigen Fällen gewollt steuernde Wirkung entfaltete. Die Gesetzesvorgaben zur Umgestaltung des Vergütungssystems griffen dies insofern auf, als dass 
- die bisherige Budgetierung gelockert wurde, indem nicht länger der Grundlohnsummenanstieg Maßstab für die Entwicklung des intrabudgetären Bereichs der Gesamtvergütung sein sollte, sondern die "Morbiditätsbedingung «: über diagnosebezogene Anpassungskriterien wird der Teil an Leistungsmehrbedarfen finanziert, der auf eine tatsächliche Erhöhung der Krankheitslast zurückzuführen ist,

- das Morbiditätsrisiko (Krankheitsrisiko der Bevölkerung und die damit verbundenen finanziellen Lasten) von den Ärzten auf die Krankenkassen zu verlagern war. Diese sollten dann auch nur solche Mehrbedarfe an vertragsärztlichen Leistungen zusätzlich vergüten, welche auf eine tatsächliche Erhöhung der bevölkerungsbezogenen Krankheitslast zurückzuführen und medizinisch notwendig sind, nicht jedoch angebotsseitig induzierte Leistungsausdehnungen bedeuten,

- eine Gebührenordnung mit festen Preisen in Euro geschaffen und die Mengensteuerung über Regelleistungsvolumina (RLV) vereinheitlicht wurde, um Verteilungsgerechtigkeit zwischen den Ärzten (arztseitige Vergütung) zu gewährleisten,

- die Krankenkassen bei der Zahlung der Gesamtvergütungen (kassenseitige Vergütung) gleichbehandelt werden sollten, indem die kassenspezifischen Gesamtvergütungen zumindest für den Übergang von 2008 auf 2009 wieder an die aktuellste historische Leistungsinanspruchnahme der Versicherten der jeweiligen Kasse gekoppelt wurde.

Vor allem aber wurden die Entscheidungskompetenzen des (Erweiterten) Bewertungsausschusses auf der Bundesebene ausgebaut, während die Spielräume der Landesebene deutlich verringert wurden. Die Ermittlung wie auch Verteilung der Gesamtvergütungen sollte fortan zentral vorgegeben werden.

\section{Erfahrungen - Hat sich das neue System bewährt?}

Obgleich im dritten Jahr seit der Einführung der morbiditätsbedingten Gesamtvergütung die Anwendung vereinbarter Kodierrichtlinien in der Praxis aussteht und die vertragsärztliche Vergütung dennoch zwischen 2007 und
2010 insgesamt um fast 5 Mrd. Euro (ca. $+18 \%$ ) angestiegen ist, hat das neue System der vertragsärztlichen Vergütung manchen Bekundungen nach auch Enttäuschungen hervorgerufen:

- Die zentral im Bewertungsausschuss auf Bundesebene geregelte Honorarverteilung wird vor Ort als zu kompliziert empfunden, die Honorarbescheide der KVen sind für die meisten Ärzte nicht nachvollziehbar (Intransparenz),

- Durch die regional unterschiedliche Leistungsmengendynamik setzt erneut eine Spreizung der tatsächlich ausgezahlten Punktwerte ein (Preisungleichheit),

- Zwischen und innerhalb von KVen und Arztgruppen bestehen weiterhin erhebliche Vergütungsunterschiede, sodass trotz der erheblich gestiegenen Vergütung eine gefühlte Unterfinanzierung besteht (Verteilungsungerechtigkeit),

- Kalkulationsmängel im Einheitlichen Bewertungsmaßstab, insbesondere eine Überbewertung der Praxiskosten, privilegieren systematisch Leistungen mit einem höheren technischen Anteil und sanktionieren die "sprechende Medizin «.

Proteste ob gefühlter Ungerechtigkeiten sowie neben schweigenden Gewinnern auch allem Anschein nach Verlierer der Honorarreform haben den Gesetzgeber bewogen, mit dem durch das GKV-Finanzierungsgesetz (GKV-FinG) neu eingefügten $\mathbb{S} 87$ Abs. 9 SGB V eine Überprüfung und Korrektur der Honorarreform in Aussicht zu stellen. Ziel war eine Konvergenz der Vergütungen und ein einfaches, verständliches Vergütungssystem. Des Weiteren sollten im Rahmen des Versorgungsgesetzes die Rahmenbedingungen für die Verteilung der Honorare auf die Ärzte verändert werden.

Der Bewertungsausschuss wurde beauftragt, bis zum 30. April 2011 ein Konzept zur schrittweisen Konvergenz zu erstellen, konnte die gesetzte Frist jedoch nicht einhalten. Die erbetene Nachfrist bis zum 20. Juni 2011 wurde vom neuen Gesetzgebungsverfahren eingeholt.

\section{Konvergenz des GKV- Finanzierungsgesetzes - Kurzes Gastspiel eines Irrlichts}

Der Kabinettsentwurf zum Versorgungsstrukturgesetz zeigt, dass die Politik das Votum der Selbstverwaltung hinsichtlich der Ausgestaltung des Konvergenzparagraphen nicht abzuwarten bereit ist und stattdessen die Korrektur der Honorarreform selbst in die Hand nimmt. Kurioserweise finden sich Bestimmungen zu einer Konvergenz der Vergütungen darin nicht wieder. Im Gegenteil - durch die weitgehende Entmachtung des Bewertungsausschusses und die Regionalisierung der Honorarverhandlungen werden allen interregionalen Angleichungsbestrebungen faktisch die Grundlage entzogen.

Und das ist auch gut so. Der Idee der Konvergenz der Vergütungen fehlte von Beginn an eine nachvollziehbare Begründung und jedwede Aussicht auf Er-

\section{Der Idee der Konvergenz der Vergütungen fehlte von Beginn an eine nachvollziehbare Begründung}

folg, die Verteilungskämpfe zwischen den Regionen nachhaltig zu befrieden.

Ausgangspunkt des Konvergenzgedankens waren die während der letzten Honorarreform transparent gewordenen Unterschiede in den von den Krankenkassen je Versicherten gezahlten durchschnittlichen Vergütungen in den KV-Bezirken. So zahlen die Krankenkassen für einen in Berlin wohnenden GKV-Versicherten 19\% (2011) mehr als für ihre Versicherten mit Wohnsitz in Sachsen-Anhalt. Dieser auf den ersten Blick erstaunliche Befund ist jedoch die unmittelbare Konsequenz aus der im Rahmen der Honorarreform vorgenommenen Ableitung der Vergütung aus der jeweiligen historischen Leistungsinanspruchnahme und damit aus dem morbiditätsbedingten Behandlungsbedarf der jeweiligen Versicherten. Die Budgets bemessen sich also an den Leistungen, die von den Ärzten tatsächlich abgerechnet worden sind. Der Gleichschritt von Leistungsmenge und Vergütung zumindest in der Stunde Null der Honorarreform gewährleistete ein vergleichbares Preisniveau zwischen den Regionen. 
Im Rahmen der Konvergenzdiskussion wurden viele Modelle gehandelt und wieder verworfen. Der Vorschlag der Kassenseite hatte neben einer Ankoppelung der von den Krankenkassen zu zahlenden Vergütungen an die jeweils aktuellste (historische) Leistungsinanspruchnahme ihrer Versicherten die Angleichung der je Leistungseinheit gezahlten Punktwerte bzw. Preise auf regionaler und Kassenebene zum Ziel.

Im Gegensatz dazu stehen Vorschläge, die eine Konvergenz auf Ebene des durchschnittlichen Behandlungsbedarfs je Versicherten vorsehen. Diesen ist gemein, dass sie die von den Krankenkassen zu zahlenden Vergütungen vom tatsächlichen Leistungsgeschehen abkoppeln. Als Folge träten Verwerfungen bei den Preisen auf: In einem Teil der KVen würden künstlich erhöhte Behandlungsbedarfe und damit Zahlbeträge auf relativ niedrige tatsächlich erbrachte Leistungsmengen treffen, womit der Abrechnungspunktwert in die Höhe getrieben würde.

Bei dem von der Kassenärztlichen Bundesvereinigung (KBV) favorisierten Modell, dass sich in seiner Konstruktion deutlich an die Methodik des morbiditätsorientierten Risikostrukturausgleichs anlehnt, kommt hinzu, dass es die Zentralisierung der Vergütungsfindung auf die Spitze getrieben hätte. Eine zentrale $\mathrm{Zu}$ weisung von KV- bzw. kassenbezogenen Vergütungsbeträgen anhand von diagnosebezogenen Normwerten schließt regionale Verhandlungsspielräume aus. Nicht zuletzt deshalb und aufgrund der zu erwartenden Umverteilungswirkungen hatte eine Mehrheit der KVen dieses Modell erst einmal gestoppt. Denn von vorneherein war allen Beteiligten klar, dass eine konvergenzbedingte Neu- und Umverteilung der Vergütungen nicht vollständig durch Mehrzahlungen der Krankenkassen kompensiert würde. Für einige KVen wäre eine Konvergenz also mit realen Vergütungsverlusten verbunden gewesen. Mit dem Kabinettsentwurf sind derartige Gedankenspiele vorläufig vom Tisch.

\section{Die Honorarreform des Versorgungsstrukturgesetzes - Regionalisierung und Monopolisierung}

Ein leistungsgerechtes und einfaches Vergütungssystem zu schaffen war ein
Ziel der Konvergenz, das auch nach deren Untergang aktuell bleibt und weiter anzustreben wäre. Allerdings weist der Kabinettsentwurf in eine andere Richtung.

Die Neufassung der $\mathbb{S} \int 87,87 \mathrm{a}$ bis c SGB V lässt sich in ihrem Kern mit den Begriffen Regionalisierung und Monopolisierung beschreiben. Regionalisierung, weil zukünftig die wesentlichen Entscheidungen zu Honorarverhandlung und -verteilung auf der Ebene der KV-Bezirke getroffen werden anstatt im Bewertungsausschuss. Monopolisierung, weil die Verteilung von Honorar und Information in das nahezu alleinige Ermessen der KVen und der KBV gestellt werden. Die Rolle der Krankenkassen und ihrer Verbände wird entweder auf Null reduziert (Honorarverteilung und Datentransparenz), beschränkt sich auf die Mitwirkung an Empfehlungen (Bewertungsausschuss) oder besteht in der regionalen Verhandlungspartnerschaft, deren tatsächlicher Wert aber maßgeblich davon abhängen wird, inwieweit sich die den Kassen zur Verfügung stehenden Datengrundlagen verbessern.

Die Regionalisierung der Ressourcenentscheidungen kann sachgerecht sein, ist aber für sich allein genommen noch kein Problemlöser. So ist die geplante Stärkung der regionalen Ausgestaltung der Honorarverteilung grundsätzlich begrüßenswert, da hier regionale Besonderheiten in der Angebots- und Leistungsstruktur eine hohe Bedeutung haben. Die derzeitige Kombination aus einem bundeseinheitlichen, unabdingbaren Regelungskern und einer Vielzahl dispositiver Vorgaben hat sich nicht bewährt. Die Vorstellung einer transparenten und zwischen den KV-Bezirken vergleichbaren Honorarverteilung, die noch dem GKV-WSG mit seiner Rollenzuweisung an den Bewertungsausschuss zugrunde lag, ist der Realität eines unermüdlichen Reparaturbetriebs gewichen, in dem der Bewertungsausschuss seine eigenen Beschlüsse im Monatsrhythmus korrigiert hat und die Partner der Gesamtverträge zwang, ihre Vereinbarungen ständig anzupassen. Selbst tiefgreifende Strukturveränderungen wie die Einführung der Qualifikationsbezogenen Zusatzvolumina erfolgten hastig und im Affekt von vermuteten Fehlent- wicklungen, bevor eine belastbare empirische Evidenz vorlag.

Bei aller berechtigten Kritik am Bewertungsausschuss besteht aber kein Anlass, sein Heil im entgegengesetzten Extrem der totalen Regionalisierung zu suchen. Es gibt gute Gründe dafür - und dazu ist nicht zuletzt die Gewährleistung einheitlich anzuwendender, verlässlicher und planungssicherer Bereinigungsregeln für die arztseitige Vergütung zu zählen -, dass bundesweite Rahmenvorgaben ein Mindestmaß an Standardisierung und Vergleichbarkeit sicherstellen. Erstaunlicherweise sehen gerade die KVen mehrheitlich den Bedarf, die Trennung der Haus- und fachärztlichen Vergütungsbereiche bundeseinheitlich und einvernehmlich im Bewertungsausschuss regeln zu lassen. Daher kommt es auf die richtige Balance an, wie ein bundesweiter Rahmen und der regionale Regelungskern gegeneinander abgegrenzt werden und welche Entscheidungsfindungsprozesse zum Tragen kommen.

Eine alleinige Festlegung des Honorarverteilungsmaßstabes (HVM)

Die Regionalisierung der rcenentscheidungen kann gerecht sein, ist aber für sich allein genommen noch kein Problemlöser.

durch die KVen, wie es der Kabinettsentwurf vorsieht, würde allerdings einen Rückschritt in die Zeit vor dem Gesundheitsmodernisierungsgesetz darstellen, mit dem aus guten Gründen (z.B. Schutz kleinerer Arztgruppen) das Vertragsprinzip in die Honorarverteilung eingeführt worden war. Vertragsverhandlungen erzwingen in ihrem Wechselspiel aus Macht und Gegenmacht Kompromisse und den Ausgleich von Interessen. Für Krankenkassen stehen bei der Honorarverteilung immer deren Auswirkungen auf die Versorgung im Vordergrund. Aufgrund der Gesetzmäßigkeiten der Willensbildung innerhalb der KVen neigen diese hingegen stets zur Besitzstandswahrung, also der Fortschreibung des Status quo. Sobald die KVen autonom über die Honorarverteilung befinden können, wird dieser Interessenkonflikt im Sinne der Besitzstandswahrung entschieden. 
Denn der Kabinettsentwurf lässt es völlig offen, welchen anderen Zielen oder Grundsätzen die KVen bei der Erstellung des Honorarverteilungsmaßstabes eigentlich verpflichtet sein sollen. Daher ist nicht zu erwarten, dass die KVen in eigener Verantwortung Anstrengungen unternehmen werden, um relativieren, steht das » $M$ « vor der Gesamtvergütung endgültig zur Disposition. Diese Entwicklung ist erstaunlich, galt doch die Morbiditätsbedingung, die die ungeliebte Grundlohnsummenbindung ablöste, als die Errungenschaft ärztlicher Politikbearbeitung im sonst wenig ärztefreundlichen GKV-WSG.

Doch die Verweigerung breiter Teile der niedergelassenen

Ärzteschaft, die ambulanten Kodierrichtlinien anzuwenden, hat diesen unbestreitbaren Erfolg wieder zunichte

in punkto leistungsgerechter und einfacher Vergütung tätig zu werden. Diese Passivität würde durch die Intransparenz erleichtert, die durch das Informationsmonopol der KVen über die Abrechnungsdaten ermöglicht wird. Die Honorarverteilung würde zu einem geschlossenes System, das einer externen Überprüfung und Korrektur unzugänglich wäre.

Von daher ist es sachgerecht, die Vertragspartner in den KVen weiterhin die Honorarverteilung vereinbaren zu lassen und sie in ihren Regelungskompetenzen dahingehend zu stärken, dass auch Versorgungsgesichtspunkte Gegenstand der Honorarverteilung sind. Dies schließt eine Ausweitung der Möglichkeiten der regionalen Vertragspartner zur Versorgungsforschung und -analyse im Sinne einer effektiveren Datenverfügbarkeit und -nutzung für alle Vertragspartner mit ein. Die Feststellung, welche Daten zur vertragsärztlichen Vergütung veröffentlicht werden und welche nicht, kann nicht autonome Entscheidung der einzelnen KVen oder der KBV sein.

\section{Die morbiditätsbedingte Gesamtvergütung - der Abstieg eines Superstars}

Während das Versorgungsstrukturgesetz der Konvergenz offenbar ein schnelles Ende bereitet, setzt die Politik bei dem Konzept der morbiditätsbedingten Gesamtvergütung offenbar auf eine Taktik des langen Siechtums. Mit dem Ende der Kodierrichtlinien und der daraus folgenden Entscheidung im Kabinettsentwurf, die Verbindlichkeit der diagnosebezogenen Steigerungsrate zu gemacht. Ohne Kodierrichtlinien kann es keinen Diagnosebezug in der vertragsärztlichen Vergütung geben. Der Kabinettsentwurf hat dieser Einsicht, wenn auch nur halbherzig, Rechnung tragen wollen und nun fließen Diagnosen und Demographie parallel in die Honorarverhandlung ein - in welchem Verhältnis, soll den regionalen Vertragspartnern überlassen bleiben.

Diese Regionalisierung der Honorarverhandlungen wird dazu führen, dass die Morbiditätsbedingung so viele Ausprägungen erhält wie es KV-Bezirke gibt. Durch die Vielfalt der Regelungen, insbesondere die Unterschiede in der Gewichtung Demographie/Diagnosen und der Berücksichtigung weiterer Morbiditätsfaktoren, wird der ohnehin dehnbare Morbiditätsbegriff in der ambulanten Vergütung vollends diffus und degeneriert zu einer Leerformel. Es wäre konsequenter und ehrlicher gewesen, diese ehemalige Ikone ärztlicher Interessenvertretung in Würde, direkt neben der Konvergenz zu beerdigen.

Inwieweit mit der Regionalisierung der Honorarverhandlungen generell ein zukunftsweisender Weg eingeschlagen wird, bleibt abzuwarten. Vielleicht gelingen den Partnern der Gesamtverträge Ergebnisse, die in höherem Maße von den tatsächlichen Versorgungsrealitäten vor Ort und weniger von interregionalen Proporz- und Verteilungsgesichtspunkten geprägt sind. Sicher ist dies jedoch nicht. Regionalisierung allein ist kein Garant für eine nachhaltige Wirkung einer Honorarreform.
Dazu müsste insbesondere dafür Sorge getragen werden, dass die benannten Fehlentwicklungen der letzten Honorarreform nicht dauerhaft zementiert werden. Hierfür ist es unter anderem notwendig, dass eine stetige Anpassung der auf regionaler und Kassenebene je Leistungseinheit tatsächlich gezahlten Punktwerte erreicht wird. Leitgedanke dieses Ansatzes ist eine "gleiche Vergütung für gleiche bzw. vergleichbare Leistung «. Hierbei soll im Binnenverhältnis der Krankenkassen und, wenn möglich, auch der Ärzte untereinander der gleiche Preis je erbrachter Leistungseinheit nach EBM vergütet werden, und zwar für alle gleichermaßen proportional zur Leistungsinanspruchnahme nach EBM der Versicherten. Um der regional und fachgruppenspezifisch unterschiedlichen Mengendynamik zu begegnen, die Überbewertung technischer Leistungen $\mathrm{zu}$ beenden und einen Mengenwettlauf zu verhindern, ist eine ohnehin überfällige (gesetzlich nur als Kann-Regelung vorgegebene) Neuordnung und -kalkulation des EBM erforderlich.

Dadurch, dass sich die Krankenkassen an der Vergütung des KV-bezogenen Behandlungsbedarfs anteilmäßig entsprechend der relativen durchschnittlichen Leistungsinanspruchnahme ihrer jeweiligen Versicherten beteiligen, können die Punktwerte zwischen den Kassen
Ohne Kodierrichtlinien kann es keinen Diagnosebezug in der vertragsärztlichen Vergütung geben.

einheitlich gehalten und somit Wettbewerbsverzerrungen vermieden werden.

\section{Defizite der vertragsärztlichen Vergütung werden nicht angesprochen}

Denn die Ursachen der permanenten inneren Unzufriedenheit, in der sich die Vertragsärzte bewegen, sind weniger bei den behaupteten Vergütungsunterschieden zwischen den KVen zu suchen als in der innerärztlichen Verteilung von Honorar und Einkommen (bei gegebener Leistung). Die durchschnittlichen Praxiserträge, also die GKV-Honorare abzüglich der Praxiskosten, weichen zwischen 
den Arztgruppen deutlich voneinander ab. Während zur Kalkulation des EBM für jeden Arzt ein einheitlicher kalkulatorischer Arztlohn herangezogen wurde, weist die Realität erhebliche Einkommensunterschiede (wiederum bei gegebener Leistung) aus.

Diese Unterschiede waren und sind nicht gewollt. Kein Gremium der Selbstverwaltung und keine Rechtsnorm haben sie beabsichtigt oder festgelegt. Sie sind Begleiterscheinungen von Kalkulationsmängeln im EBM, der aufgrund einer systematischen Überschätzung der Praxiskosten technische Leistungen überbewertet. Spätere Honorarverteilungsmaßstäbe, die die Fachgruppenbudgets nach den jeweiligen Anteilen an der abgerechneten Leistungsmenge bemaßen, ließen diese Überbewertungen honorarwirksam werden.

Diese schleichende Verschiebung von Honorarmitteln $\mathrm{zu}$ technisch orientierten Fachgruppen hat Folgen. Wo es an einer leistungsgerechten Vergütung fehlt oder zu fehlen scheint, drängen Ärzte und Arztgruppen zunehmend in privatärztliche Angebote. Bei den Hautärzten entfällt nach Erhebungen des Statistischen Bundesamtes mehr als die Hälfte des Honorars auf privatärztliche Leistungen. Bei den HNO-Ärzten wurden zu Beginn des Jahres Komfortsprechstunden als IGeL-Angebote diskutiert, die zu einer weiteren Verknappung der Sprechstundenzeiten für GKV-Versicherte führen würden. Gerade an diesen Beispielen wird deutlich, wie eng der Zusammenhang zwischen Vergütung und Versorgung, dem ursprünglichen Kern des Versorgungsstrukturgesetzes, ist.

Die Instrumente des Kabinettsentwurfs, Regionalisierung und Monopolisierung, adressieren diese Fehlentwicklungen nicht. Es wird weiteren Gesundheitsreformen vorbehalten sein, hier anzusetzen.

\section{Nach der Reform ist vor der Reform}

Es ist nicht erkennbar, dass die Regelungen zur vertragsärztlichen Vergütung mit einem übergeordneten ordnungspolitischen Ziel verbunden sind. Stattdessen scheint die Politik entschlossen, der ärztlichen Selbstverwaltung Prokura zu erteilen, damit sie in eigener Verantwortung die vertragsärztliche Vergütung organisiert.
Denn nichts anderes ist das Versorgungsstrukturgesetz als ein Auftrag an die KVen und die KBV als Interessenvertretung der Vertragsärzte: Hört auf zu jammern und macht's selbst!

Jetzt lässt es sich trefflich darüber streiten, ob derartige Reaktionen einer verantwortlichen Politik gut zu Gesicht stehen. Gemeinhin wird erwartet, dass mithilfe von Gesetzen die Problemlösung versucht und nicht lediglich delegiert wird. Insbesondere da nicht anzunehmen ist, dass das KV-System plötzlich die Befriedung der Ärzteschaft gelingt, zumal dies überhaupt nicht im Interesse der KVen liegen kann. Denn nur aus der latenten Unzufriedenheit der Ärzte kann derjenige politische Druck erzeugt werden, der den Boden für die gewünschte Honorarentwicklung bereitet.

Diese Unzufriedenheit von sich auf die ärztliche Selbstverwaltung selbst zu richten, ist daher die eigentliche Intention des Kabinettsentwurfs. Das BMG und die Regierungsfraktionen werden nicht ernsthaft damit rechnen, dass die Delegation an das KV-System tatsächlich auch nur ein vorhandenes Problem beseitigt, außer jenes, wiederholt für die Entscheidung der Selbstverwaltung in die Mitverantwortung genommen zu werden. Es mag paradox klingen, aber in der Ermächtigung des KV-Systems liegt zugleich ein Misstrauensvotum, denn sie kalkuliert das Scheitern mit ein.

Denn es ist jetzt schon absehbar, dass die KVen die an sie gesetzten Erwartungen nicht oder nur um den Preis erheblicher Honorarsteigerungen werden erfüllen können. Viele KVen haben selbst die Erwartung geweckt, in regionalen Verhandlungen bessere Ergebnisse erzielen zu können, als die KBV im Bewertungsausschuss. Letzteres wäre allerdings nur denkbar, wenn ein entsprechendes politisches Klima vorhanden ist, wie es noch 2008 der Fall war, als die KBV Rückendeckung aus dem BMG erfuhr. Ob die KVen im kommenden Jahr darauf hoffen dürfen, kann bezweifelt werden.

Aber auch dies könnte die bestehenden grundsätzlichen Probleme womöglich für einen begrenzten Zeitraum verdecken; die Reaktionen auf die zurückliegende Honorarreform zeigen, dass ohne strukturelle Eingriffe in die Honorarverteilung auch außerordentliche Honorarzuwächse verpuffen.

Das Danaergeschenk legt den Keim für die nächste Reform. Und ein Beispiel wie eine Reform der vertragsärztlichen Vergütung nach einem Scheitern der KVen in ihrer neuen Rolle, die zugleich auch ihre alte ist, aussehen könnte, liefert der Kabinettsentwurf gleich mit: der \$ 116 b SGB V in der Entwurfsfassung sei hierfür zur Lektüre empfohlen.

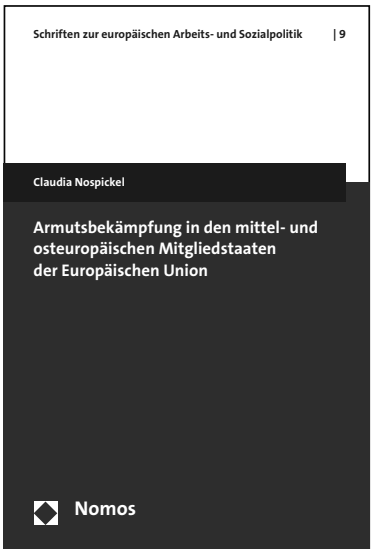

\section{Armutsbekämpfung in den mittel- und osteuro- päischen Mitgliedstaaten der Europäischen Union}

Von Dr. Claudia Nospickel 2011, ca. 350 S., brosch., ca. 69, $-€$ ISBN 978-3-8329-6577-8

(Schriften zur europäischen Arbeits- und Sozialpolitik, Bd. 9)

Erscheint ca. Oktober 2011

nomos-shop.de/13583

In der Transformationsphase der 1990er Jahre entwickelte sich Armut zu einem gravierenden Problem in den Staaten Mittel- und Osteuropas. Am Beispiel der Länder Estland, Tschechien, Polen und Ungarn zeichnet die Studie diese Entwicklung bis zum Beitritt zur EU nach und stellt die Frage, wie die neuen Mitgliedstaaten Strategien der EU zur Armutsbekämpfung aufgreifen.

\section{Nomos}

Bitte bestellen Sie im Buchhandel oder versandkostenfrei unter $\checkmark w w w$.nomos-shop.de 